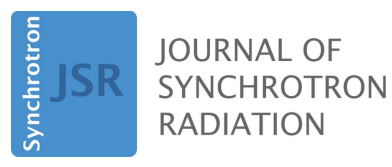

ISSN 1600-5775

\section{Third International Workshop on Improving Data Quality in XAFS Spectroscopy, Q2XAFS2017}

\author{
Sofia Diaz-Moreno ${ }^{\mathrm{a} *}$ and Richard W. Strange $\mathrm{e}^{\mathrm{b} *}$ \\ aDiamond Light Source, Harwell Campus, Didcot, Oxfordshire OX11 ODE, UK, and \\ ${ }^{\mathbf{b}}$ School of Biological Sciences, University of Essex, Wivenhoe Park, Colchester, Essex CO4 3SQ, UK. \\ *Correspondence e-mail: sofia.diaz-moreno@diamond.ac.uk, rstrange@essex.ac.uk
}

This issue of the Journal of Synchrotron Radiation is a special issue including papers from the Q2XAFS2017 workshop. Here, a brief introduction is given.

The International Workshop on Improving Data Quality in XAFS Spectroscopy, Q2XAFS2017, was held at Diamond Light Source (UK) in August 2017. The meeting was proposed by the International Union of Crystallography (IUCr) Commission on XAFS as a satellite workshop to the main IUCr Congress held in Hyderabad (India) one week after the workshop, and continues the series of successful meetings on the subject that began in Tsukuba (Japan) in 2011, and then in Hamburg (Germany) in 2015 .

X-ray absorption fine structure (XAFS) is a well established technique used for the determination of the geometrical and electronic structures of a specific element in a variety of materials. The versatility of the technique makes it suitable for the study of crystalline and amorphous solids, liquids or gases. Nowadays XAFS is used in a routine manner in many different disciplines, from chemistry and catalysis to environmental science, biology and hard condensed matter and more. Several attempts have been made in the past to put in place a set of international standards for XAFS that are similar to those established by the IUCr for protein crystallography. The International X-ray Absorption Society (IXAS) and the IUCr Commission on XAFS are actively working to achieve this goal.

Following the same rational as its predecessors, Q2XAFS2017 was organized to provide a dynamic forum for discussions between active X-ray absorption spectroscopy researchers to review international standards and protocols, and to develop revised recommendations for best practice. The workshop also aimed to establish agreed data formats and standards for XAFS data and its deposition and storage.

The workshop was organized by Prof. Christopher Chantler, chair of the IUCr Commission on XAFS, Dr Matthew Newville, Vice-chair of the executive committee of the IXAS, and Dr Sofia Diaz-Moreno, member of both the IUCr Commission on XAFS and the executive committee of the IXAS. Sixty people, half of whom were from outside the UK (USA, France, Brazil, Spain, Sweden, Italy, Australia, Korea, Japan, Germany, Switzerland, Denmark, The Netherlands and China) met over two days at Diamond Light Source (DLS) to discuss the future of XAFS spectroscopy.

The workshop was divided into six sessions comprising speaker presentations and audience discussions that covered the main aspects of XAFS that affect data quality.

The first session, chaired by Sofia Diaz-Moreno (DLS), was focused on Data Collection Optimization. Christopher Chantler (University of Melbourne) and Roberto BoadaRomero (then at DLS, now at the Autonomous University of Barcelona) spoke about instrumental aspects that need to be optimized to achieve good quality data. Giuliana Aquilanti (Synchrotrone Elettra) discussed aspects that need to be optimized when carrying out experiments under high-pressure conditions, and Ritimukta Sarangi (SSRL) gave an overview of the challenges when performing Bio-XAS.

The second session was focused on Data Analysis. It was chaired by Richard Strange (University of Essex), with two invited speakers: Matthew Newville (APS) and Daniel Bowron (ISIS). While Matt talked about recent advances in XAFS analysis using Larch, 


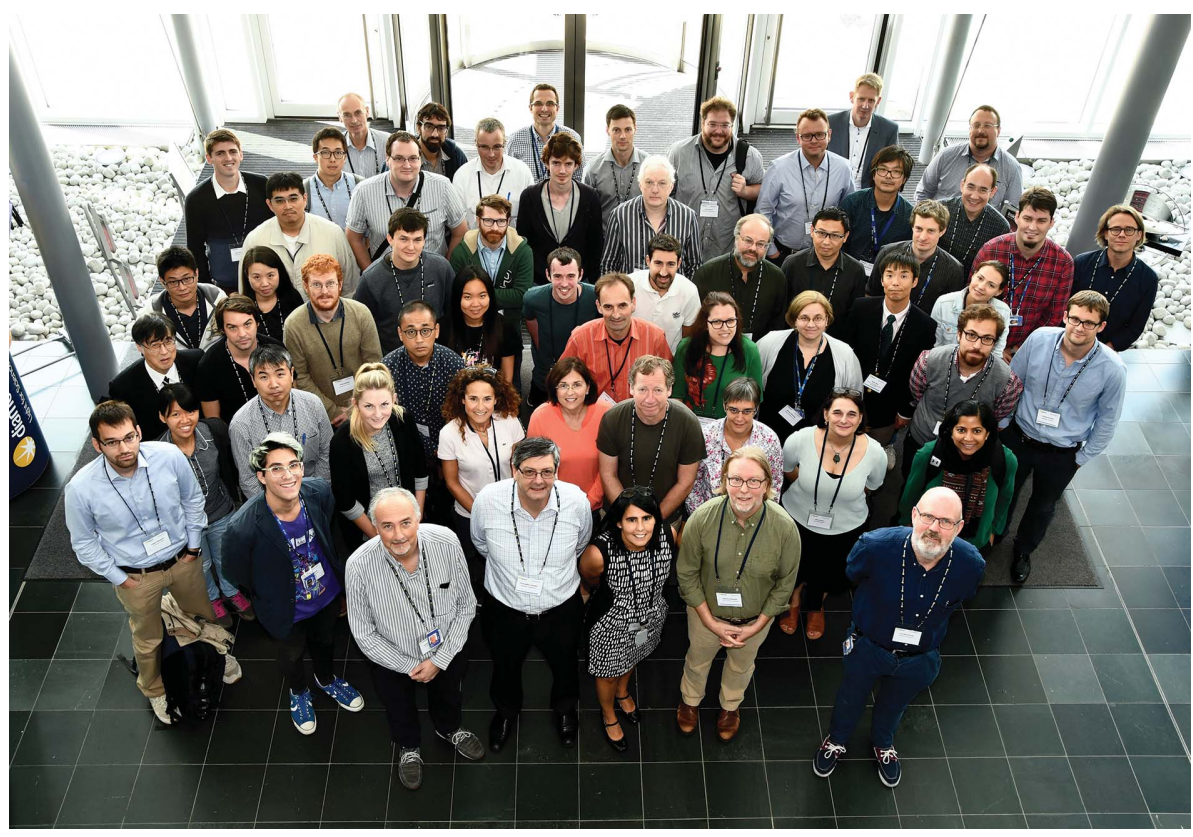

Group photograph from the Q2XAFS2017 workshop. of extending it internationally. Bruce Ravel (NIST) was the last speaker of the session and he spoke about new developments within FEFF8L.

The final session of the workshop was devoted to new experiments, and was titled Evolving Frontiers. This session was chaired by Andrew Dent (DLS), and the talks given by Stuart Bartlett (The University of Sydney), Pieter Glatzel (ESRF), Gloria Subias (ICMA) and Maarten Nachtegal (SLS) were all focused on new and challenging experiments, from pump-probe spectroscopy to XES and HR-XANES, passing through polarization-dependent XAS and XES studies and on to new challenges coming when time resolution is brought to XES.

Following the meeting, ten papers were proposed for publication and, after thorough peer review, have been collected in this issue. They cover the

Daniel spoke about combining EXAFS with diffraction data to refine comprehensive atomistic models of multicomponent glasses.

Data Quality was the subject of the third session, chaired by Christopher Chantler. Dr Edmund Welter (DESY), Bruce Bunker (University of NotreDame), Sakura Pascarelli (ESRF) and Hitoshi Abe (Photon Factory) summarized the factors affecting data quality, not only in conventional XAFS but also in the dispersive configuration of the technique. The subject of harmonic content was also discussed.

The second day of the workshop began with a session focused on Theory Developments that was chaired by Matthew Newville. Yves Joly (Institute Neel) and Thomas Penfold (University of Newcastle) gave an insight of the state-of-theart theory applied to the analysis of XANES and XES data.

The fifth session was centred on Standardization of Experiments, Automation and Data Format. The session was chaired by Fred Mosselmans (DLS). Santiago Figueroa (LNLS) and Stephen Mangold (ANKA) gave a comprehensive overview of the current automation and remote access achieved at LNLS and ANKA, while Kiyotaka Asakura (Hokkaido University) described the current status of the Japanese XAFS database and explained the advantages wide variety of topics discussed during the workshop: from error propagation and how to preserve the information content when processing XAFS data (Schalken and Chantler) to the need for establishing a methodology to benchmark XAFS measurements across laboratories (Chantler et al.); and also highlighting the importance of having standards and information exchange in Bio-XAS (Sarangi). Automation and remote access at beamlines and laboratories are covered by two of the contributions (Figueroa et al. and Mangold). The need for a XAFS database and the description of the efforts that have already been made in Japan towards achieving this goal (Asakura et al.), and the outstanding problems in XAFS instrumentation that affects data quality (Abe et al.) are both of high relevance for the XAFS community. Additional papers cover topics such as multi-technique approaches applied to glasses (Bowron), new methods in time-resolved XAFS (Guda et al.) and the state-of-the-art spectroscopy beamlines at DLS, the host of the workshop (Diaz-Moreno et al.).

You will see from the topics covered in these manuscripts that the XAFS community is moving towards implementation of internationally approved standards and protocols, and we hope that their content will help promote further discussion and agreement towards this end. 